\title{
GRAMMATISCHE KLEINIGKEITEN.
}

1. Um das $u$ in ahd. ubar neben denı in fränkischen quellen, ofters nur bei Tatian ${ }^{1}$ ) belegten obar und las $\ddot{u}$ in nhd. über zu erklären hat Joh. Schmilt in der Zschr. f. vgl. sprachforschung XXVI, 33 zu einer sehr künstlichen annabme seine zuflucht genommen: 'Das erst im hochdeutschen syncopierte $i$ der composita hatte zu der zeit, als $u$ durch folgendes $a \mathrm{zu} o$ ward, das vorhergehende $a$ schon so weit nach $i$ hin gefärbt, dass os unfähig war brechung zu bewirken; geschrieben ward es trotzdem noch mit $a$, weil dieser vocal in unbetonter silbe namentlich vor $r$ beliebt war. Als das $i$ dann schwand, hinterliess es eine mouillierung des $r$, durch welche die klangfarbe des $a$ so weit verwischt ward, dass umlaut des $u$ eintreten konnte, die schrift hielt trotzdem noch eine weile an ihm fest.' Wenn das geschwundene $i$ umlaut des $u$ hätte bewirken sollen, so hätte das auf keine andere weise geschehen können, als dass es zunächst den vocal der dazwischenliegenden silbe in ein wirkliches $i$ verwandelt hätte. Dass ein solehes, wenn es in der aussprache bestanden hïtte, durch $a$ widergegeben sein wurde, diirfte doch wol Sch. niemand glauben machon. Bekauntlich wirkt ja aber cin im ahd. gescliwundenes $i$ nicht einmal umlaut in der nächstvorhergehenden silbe, offenbar weil es keine consonantenmouillierung hinterlassen hat, wie sie für diesen fall von Sch. angenommen wird. Es ist

1) Nebenbei bemerke ich, dass es nicht zutreffend ist, wenn Schmidt in bezug auf den gebrauch von ubar und obar bei ' $T$. einen unterschied zwischen der praeposition und dem verbilen compositum statuiert. In dem letzteren erscheint obar allerdings, wie schmidt bemerkt, nur zweimal, aber ubar auch im ganzen nur sechs mal, und da auch fïr die praep. die form ubar bei weitem überwiegt, so besteht kein unterschied. 
evident, dass nbd. über nicht dem ahd. ubar, sondern nur dem ubiri ${ }^{1}$ ) entspricht. Wann die verallyemeinerung des rmlauts eingetreten ist, lässt sich mit unsern mitteln gar nicht ausmachen. Wenn man in den kritischen ausgaleu mittelhocndeutscher texte über wie im nhd. durchfuhrt, so ist das willkürlich; denn die schreibung der handschriften entscheidet nichts, und im reim kann die praeposition und das crste compositionsglied nicht vorkommen. ${ }^{2}$ ) Was dann das $u$ vor dem folgenden $a$ betriffit, so crklärt es sich daraus, dass -ar auf älteres -ur zurtackgeht (vgl. Beitr. 6, $202 \mathrm{ff}$.), welches zur zeit, als die brechung des $u$ zu $o$ eintrat, noch bestand. Gl. K. und Pa. bicten noch upur. Es eutspricht ubar dem ags. ufor, dagegen obar ${ }^{3}$ ) dem ags. ofer. Wir haben ja gerade so $u$ in sumar = ags. sumor. Vgl. auch afur neben afar.

2. Dic differenz zwischen nhd. backen und mhd. bachen wird öfters so aufyefasst, als sei das erstere eigentlich niederdeutsche form. Selbst im Dwb. wird bemerkt, dass backen so unhochdeutsch erscheine, als macken, sacken wäre. Dem hochdeutschen machen entspricht aber im nd. nicht macken, sondern maken, und backen besteht bereits im mud. (nur prat. bòken neben backede), so dass die gemination alt scin muss. Mnd. backen und mhd. bachen, ahd. bahhan entsprechen sich lautlich nicht, sondern dem ersteren nilisste md. backen, oberdeutsch, bacchen, den letzteren mud. baken entsprechen. Wir haben also für das urgermanische doppeltes neben einfachem $k$ anzusetzen. Ersteres ist auch für das oberdeutsche bezeugrt durch die schreibungen pacchet Notker, pacchen Vorauer hs., backen Berth. v. Regensb. Einfaches $k$ hat das skandinavische in libereinstinımung nit der gewöhnlichen obcrdeutschen form. Diese doppelheit greht zurlick auf einen alteren wechsel in der Hexion,

1) In ubari ist das a nach analogie von ubar eingetreten, wic in magadi neben megidi nach magad, in managì neben menigî nach manag, in gisamani neben gisemini nach saman.

2) In den niederdeutschen mundarten ist bald die form des adv. mit umlaut verallgemeinert, bald die form der praep. ohne umlaut. So heisst es mekl. $\hat{a} w r$, altmörkisch ä̈̈vr, bei Nagdeburg ewwr, dagegen göttingisch, ostfriesisch, hildesheimisch owr.

3) Vielleicht wäre die richtige entsprechung von ags. of er vielmehr ahd. *ober, und obar wäre dann eine compromissbildung, vgl. ahd. after. 
der auf mitteldeutschem gebicte noch bis ins nhd. erhalten ist. Im Dwb. wird angenerkt, dass Luther neben backen noch buch setze. Clajus gibt als normales a verbo an: backe - buch gebacken. Dasselbe tut noch Schottelius, 'Teutsche hauptsprache s. 579 und selbst Frisch in seiner bearbeitung von Bödikers grundsätzen (Berlin 1729) s. 145. In der mundart von Ruhla steht noch jetzt im praet. $c h$ neben $k$. Dem ist das mnd. backe - bôk, mnl. backe - boek unmittelbar gleich zu stellen. Es ist wol selbstverständlicb, dass dies a verbo auf ein noch älteres backu - bôk (buoch) - gibakan (gibahhan) zurlickweist. Das part. hat sich nur darum dem praes. friber angeglichen, weil der vocal gleich war, wie das part. von stantan fruher das $n$ aus dem praes. angenommen hat als das praet. (vgl. Principien der sprachgeschichte 105.6). Im oberdeutschen bat sich umgekehrt das praes. nach praet. und part. gerichtet. Das verbum gehört also zu denjenigen, welche eine eigentümliche praesensbildung bewahrt haben: $k k$ ist durch assimilation aus $k n$ oder $k w$ entstanden.

FREIBURG I/B., den 6. märz $1884 . \quad$ H. PAUL. 